Article

\title{
Generalized Hyers-Ulam Stability of Trigonometric Functional Equations
}

\author{
Elhoucien Elqorachi ${ }^{1}$ and Michael Th. Rassias ${ }^{2,3, *}$
}

1 Department of Mathematics, Faculty of Sciences, Ibn Zohr University, Agadir 80000, Morocco; elqorachi@hotmail.com

2 Institute of Mathematics, University of Zurich, $\mathrm{CH}-8057$ Zurich, Switzerland

3 Moscow Institute of Physics and Technology, 141700 Dolgoprudny, Russia; michail.rassias@math.uzh.ch

* Correspondence: michail.rassias@math.uzh.ch

Received: 18 April 2018; Accepted: 11 May 2018; Published: 18 May 2018

check for updates

\begin{abstract}
In the present paper we study the generalized Hyers-Ulam stability of the generalized trigonometric functional equations

$$
\begin{gathered}
f(x y)+\mu(y) f(x \sigma(y))=2 f(x) g(y)+2 h(y), x, y \in S \\
f(x y)+\mu(y) f(x \sigma(y))=2 f(y) g(x)+2 h(x), x, y \in S,
\end{gathered}
$$

where $S$ is a semigroup, $\sigma: S \longrightarrow S$ is a involutive morphism, and $\mu: S \longrightarrow \mathbb{C}$ is a multiplicative function such that $\mu(x \sigma(x))=1$ for all $x \in S$. As an application, we establish the generalized Hyers-Ulam stability theorem on amenable monoids and when $\sigma$ is an involutive automorphism of $S$.
\end{abstract}

Keywords: Hyers-Ulam stability; trigonometric functional equations; semigroup

\section{Introduction}

Let us consider $S$ to be a semigroup (namely a set with an associative composition), $\mu: S \longrightarrow \mathbb{C}$ to be a multiplicative function, and $\sigma: S \longrightarrow S$ to be an involutive morphism. That is, $\sigma$ is an involutive automorphism:

$$
\sigma(x y)=\sigma(x) \sigma(y) \text { and } \sigma(\sigma(x))=x \text { for all } x, y \in S
$$

or $\sigma$ is an involutive anti-automorphism:

$$
\sigma(x y)=\sigma(y) \sigma(x) \text { and } \sigma(\sigma(x))=x \text { for all } x, y \in S
$$

From the functional equation

$$
f(x y)+\mu(y) f(x \sigma(y))=2 f(x) g(y)+2 h(y), x, y \in S
$$

we can obtain several other functional equations as a special case. For example, we can deduce: The Cauchy equation

$$
f(x y)=f(x)+f(y), x, y \in S
$$

( $g=1, \mu=1, \sigma=I)$, where $I$ denotes the identity map.

The quadratic functional equation

$$
f(x y)+f(x \sigma(y))=2 f(x)+2 f(y), x, y \in S
$$


$(g=1, \mu=1, f=h)$.

Wilson's functional equation

$$
f(x y)+\mu(y) f(x \sigma(y))=2 f(x) g(y), x, y \in S
$$

$(h=0)$.

D'Alembert's functional equation

$$
f(x y)+\mu(y) f(x \sigma(y))=2 f(x) f(y), x, y \in S
$$

$(g=f, h=0)$.

D'Alembert's functional Equation (5) with $\mu=1$ is also known as the cosine functional equation and has been studied extensively for a long period of time tracing back to d'Alembert [1]. This functional equation plays a crucial role in determining the sum of two vectors in various Euclidean and non-Euclidean geometries. The continuous solutions $f: \mathbb{R} \longrightarrow \mathbb{C}$ of d'Alembert's functional Equation (5) with $\mu=1$ are known: A part from the trivial solution $f=0$, the solutions of (5) are

$$
f_{\lambda}(x)=\cos (\lambda x), \quad x \in \mathbb{R}
$$

where the parameter $\lambda$ ranges over $\mathbb{C}$ (see for example [2]).

Several authors have succeeded to determine the general solution $f: S \longrightarrow \mathbb{C}$ of d'Alembert's functional Equation (5) in the abelian as well as non abelian case. Probably the very first result obtained for a non abelian group was presented by Kannappan [3]. Under the condition that $f$ is abelian: $f(z x y)=f(z y x)$ for all $x, y, z \in S$, the solutions of the Equation (5) with $\mu=1$ are of the form

$$
f(x)=\frac{\gamma(x)+\gamma(\sigma(x))}{2}, \text { where } \gamma: S \longrightarrow \mathbb{C}
$$

is multiplicative.

In recent years, the theory of d'Alembert's functional Equation (5) with $\mu=1$ has witnessed important development. For example, for the case of non abelian groups, as shown in works by $Y$. Dilian about compact groups [4-6], Stetkær [7] for step 2-nilpotent groups, Friis [8] for results on Lie groups and Davison $[9,10]$ for general groups, even monoids.

In [11], Stetkær obtained the complex valued solutions of d'Alembert's functional Equation (5) for the case when $\mu$ is a character of the group $S$. The non-zero solutions of the Equation (5) are the normalized traces of certain representations of the group $S$ on $\mathbb{C}^{2}$

Furthermore, in [12] Ebanks and Stetkær presented some new results on groups regarding the solutions of Wilson's functional Equation (4) with $\mu=1$. We shall now also refer to Wilson's first generalization of d'Alembert's functional equation:

$$
f(x+y)+f(x-y)=2 f(x) g(y), x, y \in \mathbb{R} .
$$

The formulas constituting the solutions of this equation for the case of abelian groups are known, cf. Aczél [2], Sections 3.2.1 and 3.2.2.

In recent work, Stetkær $([13,14])$ studied the solutions of Wilson's functional Equation $(4)$ and in particular he proved that if $f, g$ are solutions of (4) with $f \neq 0$ then $g$ satisfies d'Alembert's functional Equation (5) [15]. Determining the solution formulas of $f$ is still an open problem.

In 1940, Ulam posed the stability problem for group homomorphisms [16]. The first affirmative answer to Ulam's question was presented in 1941 by Hyers [17] on Banach spaces. In 1978 Rassias [18] generalized Hyers' theorem for linear mappings by considering an unbounded Cauchy difference for sum of powers of norms. Rassias' theorem has been generalized by Gavruta [19] who allowed the Cauchy difference to be bounded by a general control function. Since that period, the corresponding 
area has become a very vibrant domain of research and stability problems for several functional equations have been extensively investigated by a number of authors (cf. [20-39]).

The stability (superstability) of d'Alembert's functional equation was first obtained by Baker [40]. Another generalization of Baker's result was presented by Székelyhidi [41]. This involves an interesting generalization of the class of bounded functions on a group or semigroup. For a series of interesting stability and superstability results, one is also reffered to the following works [20,21,42-54] Bouikhalene and Elqorachi [55] for general groups.

The generalized Hyers-Ulam stability of the functional equations (1) and

$$
f(x y)+\mu(y) f(x \sigma(y))=2 f(y) g(x)+2 h(x), x, y \in S
$$

with $\mu=1$ was studied by Badora [42] and Akkouchi [56].

A variety of stability results regarding trigonometric functional equations and their generalizations are obtained (cf. [27,57]).

The main purpose of the present paper is to study the stability of the functional Equations (1) and (6). In the sequel, we obtain some properties of the stability of Equation (1) as well as Equation (6). As an application we prove the generalized Hyers-Ulam stability of Equations (1) and (6) on amenable monoids $S$ and when $\sigma$ is an involutive automorphism of $S$.

\section{Generalized Hyers-Ulam Stability of Equation (1) on Non-Abelian Semigroups}

In the present section, we obtain properties of the stability of Equation (1).

Theorem 1. Let $\sigma: S \longrightarrow S$ be an involutive morphism of the semigroup $S$. Let $\mu: S \longrightarrow \mathbb{C}$ be a multiplicative function such that $\mu(x \sigma(x))=1$ for all $x \in S$. Suppose that the functions $f, g, h: S \longrightarrow \mathbb{C}$ satisfy the functional inequality

$$
|f(x y)+\mu(y) f(x \sigma(y))-2 f(x) g(y)-2 h(y)| \leq \phi(y)
$$

for all $x, y \in S$ and for some function $\phi: S \longrightarrow \mathbb{R}^{+}$. Under these assumptions the following statements hold: (1) If $\sigma$ is an involutive anti-automorphism and $f$ is unbounded, then $g$ is a solution of the long $d$ 'Alembert functional equation

$$
g(x y)+g(y x)+\mu(y) g(x \sigma(y))+\mu(y) g(\sigma(y) x)=4 g(x) g(y)
$$

for all $x, y \in S$.

(2) If $\sigma$ is an involutive automorphism and $f$ is unbounded, then $g$ is a solution of the short d'Alembert functional Equation (5).

Proof. (1) Let $f, g, h$ satisfy Inequality (7) with $\sigma$ an involutive anti-automorphism. Then for all $x, y, z \in S$ we have

$$
\begin{gathered}
|2 f(z)[g(x y)+\mu(y) g(x \sigma(y))+g(y x)+\mu(y) g(\sigma(y) x)-4 g(x) g(y)]| \\
=|2 f(z) g(x y)+\mu(y) 2 f(z) g(x \sigma(y))+2 f(z) g(y x)+\mu(y) 2 f(z) g(\sigma(y) x)-8 f(z) g(x) g(y)| \\
\leq|-f(z x y)-\mu(x y) f(z \sigma(y) \sigma(x))+2 f(z) g(x y)+2 h(x y)| \\
+|\mu(y)[-f(z x \sigma(y))-\mu(x \sigma(y)) f(z y \sigma(x))+2 f(z) g(x \sigma(y))+2 h(x \sigma(y))]| \\
+|-f(z y x)-\mu(y x) f(z \sigma(x) \sigma(y))+2 f(z) g(y x)+2 h(y x)| \\
+|\mu(y)[-f(z \sigma(y) x)-\mu(\sigma(y) x) f(z \sigma(x) y)+2 f(z) g(\sigma(y) x)+2 h(\sigma(y) x)]| \\
+|f(z x y)+\mu(y) f(z x \sigma(y))-2 f(z x) g(y)-2 h(y)|
\end{gathered}
$$




$$
\begin{aligned}
&+\mid \mu(x)(f(z \sigma(x) y)+\mu(y) f(z \sigma(x) \sigma(y))-2 f(z \sigma(x)) g(y)-2 h(y)) \mid \\
&+ f(z y x)+\mu(x) f(z y \sigma(x)-2 f(z y) g(x)-2 h(x) \mid \\
&+\mid \mu(y)[f(z \sigma(y) x)+\mu(x) f(z \sigma(y) \sigma(x))-2 f(z \sigma(y)) g(x)-2 h(x)] \mid \\
&+ \mid 2 g(y)[f(z x)+\mu(x) f(z \sigma(x))-2 f(z) g(x)-2 h(x) \mid \\
&+ 2 g(x)[f(z y)+\mu(x) f(z \sigma(y))-2 f(z) g(y)-2 h(y) \mid \\
&+\mid 4 g(x) h(y)+4 g(y) h(x)+2 \mu(y) h(x)-2 h(x y)-2 \mu(y) h(x \sigma(y)) \\
&- 2 h(y x)-2 \mu(y) h(\sigma(y) x)+2 h(y)+2 h(x)+2 \mu(x) h(y) \mid \\
& \leq(x y)+|\mu(y)| \phi(x \sigma(y))+\phi(y x)+|\mu(y)| \phi(\sigma(y) x)+\phi(y) \\
&+|\mu(x)| \phi(y)+\phi(x)+|\mu(y)| \phi(x)+2|g(y)| \phi(x)+2|g(x)| \phi(y) \\
&+\mid 4 g(x) h(y)+4 g(y) h(x)+2 \mu(y) h(x)-2 h(x y)-2 \mu(y) h(x \sigma(y)) \\
&-2 h(y x)-2 \mu(y) h(\sigma(y) x)+2 h(y)+2 h(x)+2 \mu(x) h(y) \mid .
\end{aligned}
$$

Since $f$ is assumed to be unbounded, then $g$ satisfies the functional Equation (8).

(2) If $\sigma$ is an involutive automorphism, then, by using Inequality (7), $\mu(x \sigma(x))=1$ and the triangle inequality, we obtain

$$
\begin{gathered}
|2 f(z)[g(x y)+\mu(y) g(x \sigma(y))-2 g(x) g(y)]| \\
\leq|2 f(z) g(x y)-f(z x y)-\mu(x y) f(z \sigma(x) \sigma(y))+2 h(x y)| \\
+|\mu(y)[2 f(z) g(x \sigma(y))-f(z x \sigma(y))-\mu(x \sigma(y)) f(z \sigma(x) y)+2 h(x \sigma(y))]| \\
+|f(z x y)+\mu(y) f(z x \sigma(y))-2 f(z x) g(y)-2 h(y)| \\
+|\mu(x)[f(z \sigma(x) y)+\mu(y) f(z \sigma(x) \sigma(y))-2 f(z \sigma(x)) g(y)-2 h(y)]| \\
\mid 2 g(y)[f(z x)+\mu(x) f(z \sigma(x))-2 f(z) g(x)-2 h(x)] \\
-2 h(x y)-2 \mu(y) h(x \sigma(y))+2 h(y)+2 \mu(x) h(y)+4 g(y) h(x) \mid \\
\leq \phi(x y)+|\mu(y)| \phi(x \sigma(y))+\phi(y)+|\mu(x)| \phi(y)+2|g(y)| \phi(x) \\
+|2 h(y)+2 \mu(x) h(y)+4 g(y) h(x)-2 h(x y)-2 \mu(y) h(x \sigma(y))| .
\end{gathered}
$$

The mapping $f$ is assumed to be unbounded, so $g$ is a solution of the short d'Alembert functional Equation (5). This completes the proof.

Theorem 2. Let $\sigma: S \longrightarrow S$ be an involutive automorphism of the amenable semigroup $S$. Let $\mu: S \longrightarrow \mathbb{C}$ be a multiplicative function such that $\mu(x \sigma(x))=1$ for all $x \in S$. Suppose that the functions $f, g, h: S \longrightarrow \mathbb{C}$ satisfy the functional inequality

$$
|f(x y)+\mu(y) f(x \sigma(y))-2 f(x) g(y)-2 h(y)| \leq \phi(y)
$$

for all $x, y \in S$ and for some function $\phi: S \longrightarrow \mathbb{R}^{+}$. Under the additionally assumption that $f$ is unbounded, there is a mapping $H: S \longrightarrow \mathbb{C}$ such that

$$
H(x y)+\mu(y) H(x \sigma(y))=2 H(x) g(y)+(1+\mu(x)) H(y)
$$

and

$$
|h(x)-H(x)| \leq \frac{1}{2} \phi(x)
$$

for all $x, y \in S$. 
Proof. For each $y$ fixed in $S$, the function

$$
x \longrightarrow f(x y)+\mu(y) f(x \sigma(y))-f(x) g(y)
$$

is bounded. Since $S$ is an amenable semigroup, then, from [58], there is an invariant mean on $B(S, \mathbb{C})$ - the space of the complex-valued bounded functions on $S$, which we denote by $m$. We can now define the following mapping $H: S \longrightarrow \mathbb{C}$ by

$$
H(x)=m\left[\frac{f_{x}+\mu(x) f_{\sigma(x)}}{2}-g(x) f\right], \quad x \in S,
$$

where $f_{x}(y)=f(y x), x, y \in S$. For all $x, y \in S$, we have

$$
\begin{aligned}
H(x y) & +\mu(y) H(x \sigma(y))=m\left[\frac{f_{x y}+\mu(x y) f_{\sigma(x) \sigma(y)}}{2}-g(x y) f\right] \\
& +\mu(y) m\left[\frac{f_{x \sigma(y)}+\mu(x \sigma(y)) f_{\sigma(x) y}}{2}-g(x \sigma(y)) f\right] .
\end{aligned}
$$

From Theorem 2 (2), $g$ is a solution of the short d'Alembert functional Equation (5), so we obtain

$$
\begin{gathered}
H(x y)+\mu(y) H(x \sigma(y))=m\left[\frac{f_{x y}+\mu(x y) f_{\sigma(x) \sigma(y)}}{2}+\frac{\mu(y) f_{x \sigma(y)}+\mu(x) f_{\sigma(x) y}}{2}-2 g(x) g(y) f\right] \\
\left.=m\left[\frac{\left(f_{y}+\mu(y) f_{\sigma(y)}\right)_{x}}{2}-g(y) f_{x}\right]+\mu(x)\left[\frac{\left(f_{y}+\mu(y) f_{\sigma(y)}\right)_{\sigma(x)}}{2}-g(y) f_{\sigma(x)}\right)\right] \\
\left.\quad+2 g(y)\left[\frac{f_{x}+\mu(x) f_{\sigma(x)}}{2}-g(x) f\right]\right] \\
=m\left[\frac{f_{y}+\mu(y) f_{\sigma(y)}}{2}-g(y) f\right]_{x}+\mu(x) m\left[\frac{f_{y}+\mu(y) f_{\sigma(y)}}{2}-g(y) f\right]_{\sigma(x)} \\
\quad+2 g(y) m\left[\frac{f_{x}+\mu(x) f_{\sigma(x)}}{2}-g(x) f\right] \\
=H(y)+\mu(x) H(y)+2 g(y) H(x)=(1+\mu(x)) H(y)+2 g(y) H(x) .
\end{gathered}
$$

Now, by using the definition of $H$, Inequality (9) and the definition of $m$, we obtain

$$
\begin{gathered}
|h(y)-H(y)|=\frac{1}{2}\left|m\left[f_{y}+\mu(y) f_{\sigma}(y)-2 g(y) f\right]-2 h(y)\right| \\
\leq \frac{1}{2} \sup _{x \in S}|f(x y)+\mu(y) f(x \sigma(y))-2 g(x) f(y)-2 h(y)| \leq \frac{1}{2} \phi(y) .
\end{gathered}
$$

for all $y \in S$. This completes the proof.

Theorem 3. Let $M$ be a monoid (a semigroup with identity element e). Let $\sigma: M \longrightarrow S$ be an involutive automorphism of the amenable monoid $M$. Let $\mu: S \longrightarrow \mathbb{C}$ be a multiplicative function such that $\mu(x \sigma(x))=1$ for all $x \in M$. Suppose that the functions $f, g, h: S \longrightarrow \mathbb{C}$ satisfy the functional inequality

$$
|f(x y)+\mu(y) f(x \sigma(y))-2 f(x) g(y)-2 h(y)| \leq \phi(y)
$$

for all $x, y \in M$ and for some function $\phi: S \longrightarrow \mathbb{R}^{+}$. Under the additionally assumption that $f$ is unbounded, there are mappings $F, H: M \longrightarrow \mathbb{C}$ such that

$$
H(x y)+\mu(y) H(x \sigma(y))=2 H(x) g(y)+(1+\mu(x)) H(y)
$$




$$
\begin{gathered}
F(x y)+\mu(y) F(x \sigma(y))=2 F(x) g(y)+(1+\mu(x)) H(y) \\
g(x y)+\mu(y) g(x \sigma(y))=2 g(x) g(y) \\
|h(x)-H(x)| \leq \frac{\phi(x)}{2}
\end{gathered}
$$

and

$$
|f(x)+\mu(x) f(\sigma(x))-2 F(x)| \leq 2 \phi(x)
$$

for all $x, y \in M$.

Proof. From Theorem 2, there is an $H: M \longrightarrow \mathbb{C}$ such that

$$
H(x y)+\mu(y) H(x \sigma(y))=2 H(x) g(y)+(1+\mu(x)) H(y)
$$

and

$$
|h(x)-H(x)| \leq \frac{\phi(x)}{2}
$$

for all $x, y \in M$. By replacing $x$ by $e$ in Inequality (12), we obtain

$$
|f(y)+\mu(y) f(\sigma(y))-2 f(e) g(y)-2 h(y)| \leq \phi(y)
$$

for all $y \in M$. If we set $F=f(e) g+H$, we obtain

$$
\begin{gathered}
|f(x)+\mu(x) f(\sigma(x))-2 F(x)| \leq|f(x)+\mu(x) f(\sigma(x))-2 f(e) g(x)-2 h(x)|+|2 h(x)-2 H(x)| \\
\leq \phi(x)+\phi(x)=2 \phi(x)
\end{gathered}
$$

for all $x \in M$.

On the other hand, we have

$$
\begin{aligned}
& F(x y)+\mu(y) F(x \sigma(y))=c \sigma[g(x y)+\mu(y) g(x \sigma(y))]+[H(x y)+\mu(y) H(x \sigma(y)) \\
= & 2 f(e) g(x) g(y)+2 H(x) g(y)+(1+\mu(x)) H(y)=2(F(x) g(y)+(1+\mu(x) H(y)
\end{aligned}
$$

for all $x, y \in M$. This completes the proof.

\section{Generalized Hyers-Ulam Stability of Equation (6) on Non-Abelian Semigroups}

In this section, we obtain the stability of Equation (6) on an amenable monoid.

Theorem 4. Let $\sigma: S \longrightarrow S$ be an involutive automorphism of the semigroup $S$. Let $\mu: S \longrightarrow \mathbb{C}$ be a bounded multiplicative function such that $\mu(x \sigma(x))=1$ for all $x \in S$. Suppose that the functions $f, g, h: S \longrightarrow \mathbb{C}$ satisfy the functional inequality

$$
|f(x y)+\mu(y) f(x \sigma(y))-2 f(y) g(x)-2 h(x)| \leq \phi(x)
$$

for all $x, y \in S$ and for some function $\phi: S \longrightarrow \mathbb{R}^{+}$. Under the additionally assumption that $f$ is unbounded, $g$ is a solution of the short d'Alembert functional Equation (5).

Proof. By using Inequality (18), $\mu(x \sigma(x))=1$, and $\sigma$ an involutive automorphism, we obtain

$$
\begin{gathered}
|2 f(z)[g(x y)+\mu(y) g(x \sigma(y))-2 g(x) g(y)]| \\
\leq|2 f(z) g(x y)-f(x y z)-\mu(z) f(x y \sigma(z))+2 h(x y)| \\
+|\mu(y)[2 f(z) g(x \sigma(y))-f(x \sigma(y) z)-\mu(z) f(x \sigma(y) \sigma(z))+2 h(x \sigma(y))]|
\end{gathered}
$$




$$
\begin{gathered}
+\mid f(x y z)+\mu(y z) f(x \sigma(y z))-2 f(y z) g(x)-2 h(x) \\
+\mu(z)[f(x y \sigma(z))+\mu(y \sigma(z)) f(x \sigma(y) z)-2 f(y \sigma(z)) g(x)-2 h(x)] \\
+\mid 2 g(x)[f(y z)+\mu(z) f(y \sigma(z))-2 f(z) g(y)-2 h(y)] \\
-2 \mu(y) h(x \sigma(y))+2 h(x)+2 \mu(z) h(x)+4 g(x) h(y)-2 h(x y) \mid \\
\leq \phi(x y)+\phi(x)+|\mu(z)| \phi(x)+|\mu(y)| \phi(x \sigma(y))+2|g(x)| \phi(y) \\
+|-2 h(x y)-2 \mu(y) h(x \sigma(y))+2 h(x)+2 \mu(z) h(x)+4 g(x) h(y)|
\end{gathered}
$$

for all $x, y, z \in S$. The mapping $f$ is assumed to be unbounded and $\mu$ is bounded, so $g$ is a solution of the short d'Alembert functional Equation (5). This completes the proof.

Theorem 5. Let $\sigma: S \longrightarrow S$ be an involutive automorphism of the amenable semigroup $S$. Let $\mu: S \longrightarrow \mathbb{C}$ be a bounded multiplicative function such that $\mu(x \sigma(x))=1$ for all $x \in S$. Suppose that the functions $f, g, h: S \longrightarrow \mathbb{C}$ satisfy the functional inequality

$$
|f(x y)+\mu(y) f(x \sigma(y))-2 f(x) g(y)-2 h(y)| \leq \phi(y)
$$

for all $x, y \in S$ and for some function $\phi: S \longrightarrow \mathbb{R}^{+}$. Under the additionally assumption that $f$ is unbounded, there is a mapping $H: S \longrightarrow \mathbb{C}$ such that

$$
H(x y)+\mu(y) H(x \sigma(y))=2 H(y) g(x)+2 H(x)
$$

and

$$
|h(x)-H(x)| \leq \frac{1}{2} \phi(x)
$$

for all $x, y \in S$.

Proof. For a mapping $l: S \longrightarrow \mathbb{C}$, we define the new functions ${ }_{x} l$ and $l^{\mu}$ by ${ }_{x} l(y)=l(x y)$ and $l^{\mu}(x)=\mu(x) l(\sigma(x))$ for all $x, y \in S$.

From Inequality (19) for each fixed $x$ in $S$, the function

$$
\frac{x f+(x f)^{\mu}}{2}-f g(x)
$$

is bounded. Since, $S$ is amenable semigroup, then there is an invariant mean $m$ on $B(S, \mathbb{C})$. By replacing $m$ by $M$ with $M(l)=m\left(l^{\mu}\right)$, we can choose $m$ such that $m\left(l^{\mu}\right)=m(l)$ for all $l \in B(S, \mathbb{C})$.

The following mapping

$$
H(x)=m\left[\frac{{ }_{x} f+\left({ }_{x} f\right)^{\mu}}{2}-g(x) f\right], \quad x \in S
$$

is well defined on $S$.

On the other hand, we obtain

$$
\begin{gathered}
\mu(y)\left({ }_{x \sigma(y)} f\right)^{\mu}(z)=\mu(y) \mu(z)_{x \sigma(y)} f(\sigma(z)) \\
=\mu(y) \mu(z) f(x \sigma(y) \sigma(z))=\mu(y z) f(x \sigma(y z))=\mu(y z)_{x} f(\sigma(y z)) \\
=\left({ }_{x} f\right)^{\mu}(y z)=\left({ }_{y}\left({ }_{x} f\right)^{\mu}\right)(z),
\end{gathered}
$$

which implies that

$$
\mu(y)\left(_{x \sigma(y)} f\right)^{\mu}={ }_{y}(x f)^{\mu}
$$


for all $x, y \in S$.

$$
(x y)^{\mu}(z)=\mu(z)_{x y} f(\sigma(z))=\mu(z) f(x y \sigma(z))=\mu(z)_{y}(x f)(\sigma(z)) .
$$

Therefore, we have $\left({ }_{x y} f\right)^{\mu}=\left({ }_{y}\left({ }_{x} f\right)\right)^{\mu}$ for all $x, y \in S$.

$$
\begin{gathered}
\mu(y)\left(_{x \sigma(y)} f\right)^{\mu}(z)=\mu(y) \mu(z) f(x \sigma(y) \sigma(z)) \\
=\mu(y z) f(x \sigma(y z))=\mu(y z)_{x} f(\sigma(y z))=\left({ }_{x} f\right)^{\mu}(z) .
\end{gathered}
$$

Therefore, we have $\left.\mu(y){ }_{x \sigma(y)} f\right)^{\mu}={ }_{y}\left({ }_{x} f\right)^{\mu}$ for all $x, y \in S$.

By using the definition of $H$, we obtain

$$
\begin{gathered}
H(x y)+\mu(y) H(x \sigma(y))=m\left[\frac{x y f+(x y f)^{\mu}}{2}-g(x y) f\right] \\
+\mu(y) m\left[\frac{\left.x \sigma(y) f+{ }_{x \sigma(y)} f\right)^{\mu}}{2}-g(x \sigma(y)) f\right] .
\end{gathered}
$$

From Theorem 4, $g$ is a solution of the short d'Alembert functional Equation (5). Since $m$ is additive, by using the above relations, we obtain

$$
\begin{gathered}
H(x y)+\mu(y) H(x \sigma(y))=m\left[\frac{x y f+\left({ }_{x y} f\right)^{\mu}}{2}-2 g(x) g(y) f\right] \\
+\left[\mu(y) \frac{x \sigma(y) f+\mu(y)\left(_{x \sigma(y)} f\right)^{\mu}}{2}\right]=m\left[\left[\frac{y\left({ }_{x} f\right)+y((x f))^{\mu}}{2}-{ }_{y} f g(x)\right]\right. \\
+\left[\frac{\mu(y)_{x \sigma(y) f}+\left({ }_{y}(x f)\right)^{\mu}}{2}-\left({ }_{y} f\right)^{\mu} g(x)\right]+2 g(x)\left[\frac{y f+\left({ }_{y} f\right)^{\mu}}{2}-f g(y)\right] \\
=m\left[\left[\frac{y(x f)+{ }_{y}\left(\left({ }_{x} f\right)\right)^{\mu}}{2}-{ }_{y} f g(x)\right]+m\left[\frac{\mu(y)_{x \sigma(y)} f+\left({ }_{y}(x f)\right)^{\mu}}{2}-\left({ }_{y} f\right)^{\mu} g(x)\right]\right. \\
+2 g(x) m\left[\frac{{ }_{y} f+\left({ }_{y} f\right)^{\mu}}{2}-f g(y)\right] .
\end{gathered}
$$

Since $m$ is invariant and $m\left(l^{\mu}\right)=m(l)$ for all bounded functions $l$ on $S$, then we obtain

$$
H(x y)+\mu(y) H(x \sigma(y))=2 H(y) g(x)+2 H(x)
$$

for all $x, y \in S$.

Finally, from Inequality (19) and the definition of $H$, we have

$$
\begin{gathered}
|h(y)-H(y)|=\frac{1}{2}\left|m\left[{ }_{y} f+\left({ }_{y} f\right)^{\mu}-2 g(y) f\right]-2 h(y)\right| \\
\leq \frac{1}{2} \sup _{x \in S}|f(y x)+\mu(x) f(y \sigma(x))-2 g(y) f(x)-2 h(y)| \leq \frac{1}{2} \phi(y) .
\end{gathered}
$$

for all $y \in S$. This completes the proof.

Theorem 6. Let $M$ be a monoid. Let $\sigma: M \longrightarrow S$ be an involutive automorphism of the amenable monoid $M$. Let $\mu: S \longrightarrow \mathbb{C}$ be a bounded multiplicative function such that $\mu(x \sigma(x))=1$ for all $x \in M$. Suppose that the functions $f, g, h: S \longrightarrow \mathbb{C}$ satisfy the functional inequality

$$
|f(x y)+\mu(y) f(x \sigma(y))-2 f(y) g(x)-2 h(x)| \leq \phi(x)
$$


for all $x, y \in M$ and for some function $\phi: S \longrightarrow \mathbb{R}^{+}$. Under the additional assumption that $f$ is unbounded, there are mappings $F, H: M \longrightarrow \mathbb{C}$ such that

$$
\begin{gathered}
H(x y)+\mu(y) H(x \sigma(y))=2 H(y) g(x)+2 H(x) \\
F(x y)+\mu(y) F(x \sigma(y))=2 F(y) g(x)+2 H(x) \\
g(x y)+\mu(y) g(x \sigma(y))=2 g(x) g(y) \\
|h(x)-H(x)| \leq \frac{\phi(x)}{2}
\end{gathered}
$$

and

$$
|f(x)-F(x)| \leq \phi(x)
$$

for all $x, y \in M$.

Proof. From Theorem 4, there is a mapping $H: M \longrightarrow \mathbb{C}$ such that

$$
H(x y)+\mu(y) H(x \sigma(y))=2 H(y) g(x)+2 H(x)
$$

and

$$
|h(x)-H(x)| \leq \frac{\phi(x)}{2}
$$

for all $x, y \in M$. By setting $y=e$ in Inequality (22), we obtain

$$
|2 f(x)-2 f(e) g(x)-2 h(x)| \leq \phi(x) .
$$

Let $F=f(e) g+H$. For all $x \in M$, we have

$$
|f(x)-F(x)| \leq|f(x)-f(e) g(x)-h(x)|+|h(x)-H(x)| \leq \frac{\phi(x)}{2}+\frac{\phi(x)}{2}=\phi(x) .
$$

On the other hand, we have

$$
\begin{gathered}
F(x y)+\mu(y) F(x \sigma(y))=f(e) \sigma[g(x y)+\mu(y) g(x \sigma(y))]+[H(x y)+\mu(y) H(x \sigma(y)) \\
=2 f(e) g(x) g(y)+2 H(y) g(x)+2 H(x)=2 F(y) g(x)+2 H(x)
\end{gathered}
$$

for all $x, y \in M$. This completes the proof.

Author Contributions: Both authors contributed equally to this work.

Acknowledgments: We are pleased to express our thanks to B. Bouikhalene and J. Brzdek for reading the paper and for providing useful remarks.

Conflicts of Interest: The authors declare no conflict of interest.

\section{References}

1. D'Alembert, J. Addition au Mémoire sur la courbe que forme une corde tendue mise en vibration. Hist. Ac. Sci. Berlin, 1750, 6, 355-360.

2. Aczél, J.; Dhombres, J. Functional Equations in Several Variables with Applications to Mathematics, Information Theory and to the Natural and Social Sciences; Encyclopedia of Mathematics and its Applications; Cambridge University Press: Cambridge, UK, 1989.

3. Kannappan, P.L. The functional equation $f(x y)+f\left(x y^{-1}\right)=2 f(x) f(y)$ for groups. Proc. Am. Math. Soc. 1968, 19, 69-74.

4. Dilian, Y. Factorization of cosine functions on compact connected groups. Math. Z. 2006, 254, 655-674.

5. Dilian, Y. Functional equations and Fourier analysis. Can. Math. Bull. 2011, 56, 218. arXiv:1006.5091. 
6. Dilian, Y. Cosine functions revisited. Banach J. Math. Anal. 2011, 5, 126-130.

7. Stetkær, H. d'Alembert's and Wilson's functional equations on step 2-nilpotent groups. Aequ. Math. 2004, 67, 241-261. [CrossRef]

8. Friis, P.d.P. d'Alembertfs and Wilsonfs functional equations on Lie groups. Aequ. Math. 2004, 67, 12-25. [CrossRef]

9. Davison, T.M.K. D'Alembert's functional equation on topological groups. Aequ. Math. 2008, 76, 33-53. [CrossRef]

10. Davison, T.M.K. D'Alembert's functional equation on topological monoids. Publ. Math. Debr. 2009, 75, 41-66.

11. Stetkær, H. D'Alembert's functional equation on groups. Banach Cent. Publ. 2013, 99, 173-191. [CrossRef]

12. Ebanks, B.R. Stetkar, H. On Wilson's functional equations. Aequ. Math. 2015, 89, 339-354. [CrossRef]

13. Stetkær, H. A link between Wilson's and d'Alembert's functional equations. Aequ. Math. 2016, 90, 407-409. [CrossRef]

14. Stetkær, H. Functional Equations on Groups; World Scientific: Hackensack, NJ, USA, 2013.

15. Stetkær, H. A note on Wilson's functional equation. Aequ. Math. 2017, 91, 945-947. [CrossRef]

16. Ulam, S.M. A Collection of Mathematical Problems; Interscience Publishers: New York, NY, USA, 1961. (Problems in Modern Mathematics; Wiley: New York, NY, USA, 1964.)

17. Hyers, D.H. On the stability of the linear functional equation. Proc. Natl. Acad. Sci. USA 1941, 27, $222-224$. [CrossRef] [PubMed]

18. Rassias, T.M. On the stability of linear mapping in Banach spaces. Proc. Am. Math. Soc. 1978, 72, $297-300$. [CrossRef]

19. Gavruta, P. A generalization of the Hyers-Ulam-Rassias stability of approximately additive mappings. J. Math. Anal. Appl. 1994, 184, 431-436. [CrossRef]

20. Badora, R. On the stability of a functional equation for generalized trigonometric functions. In Functional Equations and Inequalities; Rassias, T.M., Ed.; Kluwer Academic Publishers: Dordrecht, The Netherlands, 2000; pp. 1-5.

21. Bouikhalene, B.; Elqorachi, E. Solutions and stability of a generalization of wilson's equation. Acta Math. Sci. 2016, 36, 791-801.

22. Elqorachi, E.; Manar, Y.; Rassias, T.M. Hyers-ulam stability of the quadratic functional equation. In Functional Equations in Mathematical Analysis; Rassias, T., Brzdek, J., Eds.; Springer Optimization and Its Applications; Springer: New York, NY, USA, 2012; Volume 52, pp. 97-105.

23. Brzdek, J.; Popa, D.; Rasa, I.; Xu, B. Ulam Stability of Operators; Mathematical Analysis and its Applications; Academic Press: Cambridge, MA, USA, 2018; Volume 1,

24. Forti, G.L. Hyers-Ulam stability of functional equations in several variables. Aequ. Math. 1995, 50, 143-190. [CrossRef]

25. Gajda, Z. On stability of additive mappings. Int. J. Math. Sci. 1991, 14, 431-434. [CrossRef]

26. Hyers, D.H.; Rassias, T.M. Approximate homomorphisms. Aequ. Math. 1992, 44, 125-153. [CrossRef]

27. Hyers, D.H.; Isac, G.I.; Rassias, T.M. Stability of Functional Equations in Several Variables; BirkhNauser: Basel, Switzerland, 1998.

28. Jung, S.-M. Stability of the quadratic equation of Pexider type. Abh. Math. Sem. Univ. Hamburg 2000, 70, 175-190. [CrossRef]

29. Jung, S.-M.; Sahoo, P.K. Stability of a functional equation of Drygas. Aequ. Math. 2002, 64, 263-273. [CrossRef]

30. Jung, S.-M.; Popa, D.; Rassias, M.T. On the stability of the linear functional equation in a single variable on complete metric groups. J. Glob. Opt. 2014, 59, 165-171. [CrossRef]

31. Kim, G.H. On the stability of trigonometric functional equations. Adv. Differ. Equ. 2007, 2007, 90405. [CrossRef]

32. Kim, G.H. On the stability of the Pexiderized trigonometric functional equation. Appl. Math. Comput. 2008, 203, 99-105. [CrossRef]

33. Lee, Y.-H.; Jung, S.-M.; Rassias, M.T. Uniqueness theorems on functional inequalities concerning cubic-quadratic- additive equation. J. Math. Inequal. 2018, 12, 43-61. [CrossRef]

34. Rassias, J.M. On approximation of approximately linear mappings by linear mappings. J. Funct. Anal. 1982, 46, 126-130. [CrossRef]

35. Rassias, J.M. Solution of a problem of Ulam. J. Approx. Theory 1989, 57, 268-273. [CrossRef] 
36. Rassias, T.M. On the stability of the functional equations and a problem of Ulam. Acta Appl. Math. 2000, 62, 23-130. [CrossRef]

37. Rassias, T.M.; Tabor, J. Stability of Mappings of Hyers-Ulam Type; Hardronic Press, Inc.: Palm Harbor, FL, USA, 1994.

38. Rassias, T.M. Handbook of Functional Equations; Springer Optimization and Its Applications; Springer: New York, NY, USA, 2014; Volume 95.

39. Rassias, T.M. The problem of S. M. Ulam for approximately multiplicative mappings. J. Math. Anal. Appl. 2000, 246, 352-378. [CrossRef]

40. Baker, J.A. The stability of the cosine equation. Proc. Am. Math. Soc. 1980, 80, 411-416. [CrossRef]

41. Székelyhidi, L. On a theorem of Baker, Lawrence and Zorzitto. Proc. Am. Math. Soc. 1982, 84, 95-96. [CrossRef]

42. Badora, R. Stability properties of some functional equations. In Functional Equations in Mathematical Analysis; Rassias, T., Brzdek, J., Eds.; Springer Optimization and Its Applications; Springer: New York, NY, USA, 2011; Volume 5, pp. 3-13.

43. Badora, R. On the stability of some functional equations. In Proceedings of the 10th International Conference on Functional Equations and Inequalities, Bedlewo, Poland, 11-17 September 2005; p. 130.

44. Bouikhalene, B.; Elqorachi, E. Stability of a generalization of Wilson's equation. Aequ. Math. 2016, 90, 517-525.

45. Bouikhalene, B.; Elqorachi, E.; Rassias, J.M. The superstability of d'Alembertfs functional equation on the Heisenberg group. Appl. Math. Lett. 2000, 23, 105-109. [CrossRef]

46. Brzdek, J.; Najdecki, A.; Xu, B. Two general theorems on superstability of functional equations. Aequ. Math. 2015, 89, 771-783. [CrossRef]

47. El-Fassi, I.; Brzdek, J. On the hyperstability of a pexiderized $\sigma$-quadratic functional equation on semigroups. Bull. Aust. Math. Soc. 2018. [CrossRef]

48. Elqorachi, E.; Akkouchi, M. The superstability of the generalized dAlembert functional equation. Georgian Math. J. 2003, 10, 503-508.

49. Elqorachi, E.; Redouani, A. Solutions and stability of generalized Kannappan's and Van Vleck's functional equations. Ann. Math. Sil. 2017. [CrossRef]

50. Elqorachi, E.; Manar, Y.; Rassias, T.M. Hyers-ulam stability of Wilson's functional equation. In Contributions in Mathematics and Engineering; Pardalos, P., Rassias, T., Eds.; Springer: Cham, The Natherlands, 2016; pp. 165-183.

51. Ger, R. Superstability is not natural. Rocznik Nauk.-Dydakt. Prace Mat. 1993, 159, 109-123.

52. Ger, R.; Semrl, P. The stability of the exponential equation. Proc. Am. Soc. 1996, 124, 779-787. [CrossRef]

53. Redouani, A.; Elqorachi, E.; Rassias, T.M. The superstability of d'Alembert's functional equation on step 2-nilpotent groups. Aequ. Math. 2007, 74, 226-241. [CrossRef]

54. Zeglami, D.; Charifi, A.; Kabbaj, S. On the superstability of the pexider type generalized trigonometric functional equations. Acta Math. Sci. 2014, 34, 1749-1760. [CrossRef]

55. Bouikhalene, B.; Elqorachi, E. Stability of the spherical functions. Georgian Math. J. 2016, 23, 181-190. [CrossRef]

56. Akkouchi, M.; Elqorachi, E.; Sammad, K. Hyers-ulam-rassias stability on amenable groups. In Contributions in Mathematics and Engineering; Pardalos, P., Rassias, T., Eds.; Springer: Cham, The Nethelands, 2016; pp. 377-392.

57. Jung, S.-M. Hyers-Ulam-Rassias Stability of Functional Equations in Mathematical Analysis; Hadronic Press, Inc.: Palm Harbor, FL, USA, 2003.

58. Greenleaf, F.P. Invariant Means on Topological Groups and their Applications; Van Nostrand: New York, NY, USA, 1969.

(C) 2018 by the authors. Licensee MDPI, Basel, Switzerland. This article is an open access article distributed under the terms and conditions of the Creative Commons Attribution (CC BY) license (http:// creativecommons.org/licenses/by/4.0/). 